\title{
Lithium in young open clusters
}

\author{
R. Pallavicini \\ Osservatorio Astronomico di Palermo, Italy \\ S. Randich \\ Osservatorio Astrofisico di Arcetri, Firenze, Italy
}

J. R. Stauffer

Smithsonian Astrophysical Observatory, Cambridge, MA, USA

S. C. Balachandran

Dept. of Astronomy, University of Maryland, College Park, MD, USA

\begin{abstract}
We present lithium abundances for $\sim 50$ late-type members of the $\sim 30-50$ Myr old open clusters IC 2602 and IC 2391 derived from high-resolution spectra obtained at ESO and CTIO. These data enlarge and extend to cooler temperatures previous Li surveys of these clusters by Stauffer et al. (1989) and Randich et al. (1997). We discuss the lithium vs. temperature distribution in the two clusters and we compare our results with those obtained for the Pleiades $(\sim 120 \mathrm{Myr})$. The results are discussed in the general framework of lithium depletion in young clusters, from the pre-main sequence to the age of the Pleiades.
\end{abstract}

\section{Introduction}

Young open clusters with an age of $\sim 50 \mathrm{Myr}$, intermediate between pre-main sequence (PMS) stars $(\leq 10 \mathrm{Myr})$ and older clusters like $\alpha$ Persei $(\sim 70 \mathrm{Myr})$ and the Pleiades $(\sim 120 \mathrm{Myr})$, are crucial for investigating the depletion of lithium during PMS and the early evolution on the MS (e.g. Jeffries 1999 and Pasquini 2000 for recent reviews). IC 2602 and IC 2391, with estimated ages of $\sim 30-50$ $\mathrm{Myr}$, cover precisely this age interval. We present in this paper $\mathrm{Li}$ abundances for $\sim 50$ late-type stars in these two clusters, with membership confirmed on the basis of radial velocity measurements. These new data considerably enlarge and extend to later spectral types previous Li surveys by Stauffer et al. (1989) for IC 2391 and Randich et at. (1997) for IC 2602. Only a brief summary of this work is presented here; we refer to Randich et al. (2000) for a full account.

Stauffer et al. (1997) determined radial and rotational velocities, and $\mathrm{H} \alpha$ equivalenth widths (EWs) for candidate members of IC 2391. In the present study we have used the Stauffer et al. (1997) spectra to derive also lithium abundances; in addition, we have obtained new lithium data, as well as radial 


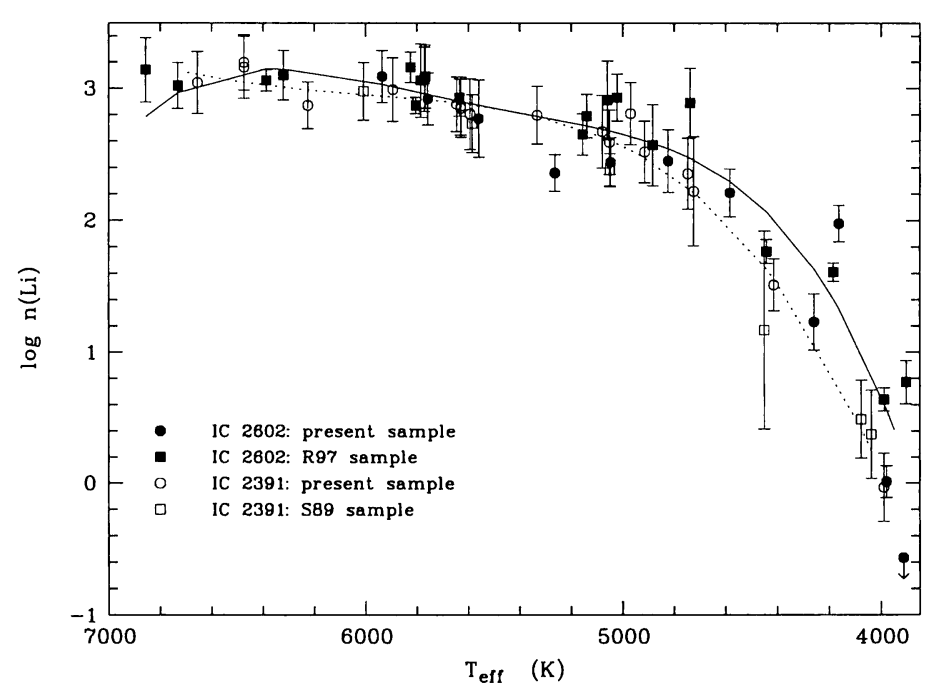

Figure 1. Li abundances vs. $\mathrm{T}_{\text {eff }}$ for IC 2602 (filled symbols) and IC 2391 (open symbols). Circles denote stars belonging to the present sample, while square represent stars in the samples of Randich et al. (1997; R97) and Stauffer et al. (1989; S89). Only stars warmer than $3800 \mathrm{~K}$ are plotted. The two curves are the regression curves of the log $\mathrm{n}(\mathrm{Li})$ vs. $\mathrm{T}_{\text {eff }}$ distributions of the two clusters (solid: IC 2602; dashed IC 2391).

and rotational velocities and $\mathrm{H} \alpha$ EWs, for a sample of IC 2602 stars. The latter sample extends to lower mass stars the previous study of IC 2602 by Randich et al. (1997). The new lithium data are combined with those of the previous surveys and compared with $\mathrm{Li}$ observations of the Pleiades. From a subsample of high S/N spectra, we have also determined for the first time the metallicity of the two clusters finding that it is nearly solar and similar to that of the Pleiades.

\section{Observations and data analysis}

The observations were obtained at the $4 \mathrm{~m}$ Blanco telescope of the Cerro-Tololo Inter-American Observatory (CTIO) in January 1995 and at the $3.6 \mathrm{~m}$ telescope of the European Southern Observatory (ESO) in April 1995.

We determined effective temperatures from the dereddened $(B-V)$ 。 and $(V-I)_{\circ}$ colors adopting the scales of Bessel (1991), Soderblom et al. (1993) and Randich et al. (1997). Lithium abundances were determined using Soderblom et al. (1993) LTE curves of growth (COGs), after correcting the measured EWs of the LiI $6707.81+\mathrm{Fe}$ I 6707.44 blend for the contribution of the Fe I 6707.44 feature. NLTE corrections were applied using the code of Carlsson et al. (1994).

Average metallicities for the two clusters were determined from the EWs of $6 \mathrm{Fe}$ I lines in the range $6700 \leq \lambda \leq 6735 \AA$ using the spectral synthesis 

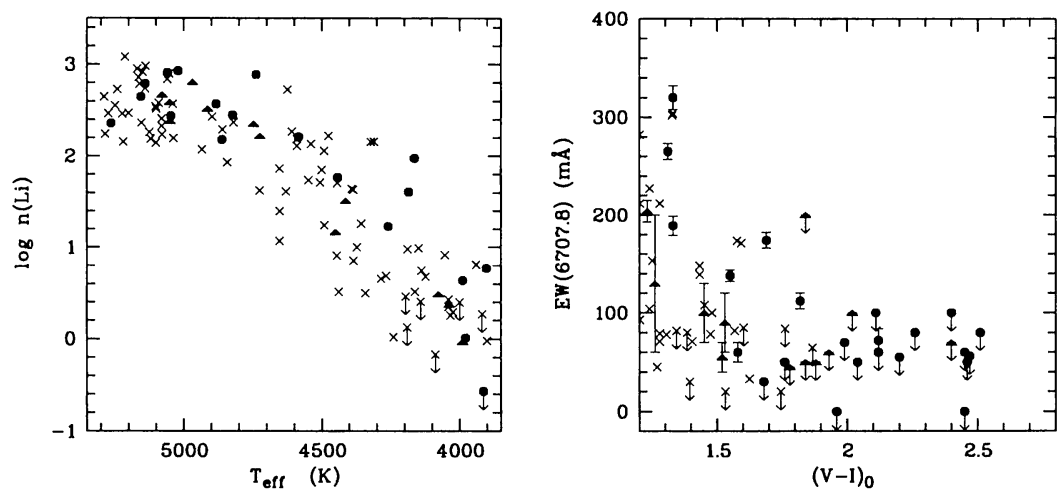

Figure 2. Comparison of the Li abundances of IC 2602 (filled circles) and IC 2391 (filled triangles) with the Pleiades (crosses). Only stars cooler than $5300 \mathrm{~K}$ are included in the figure. $\log \mathrm{n}(\mathrm{Li})$ vs. $\mathrm{T}_{\text {eff }}$ are plotted in the left-hand panel; EWs vs. $(V-I)$ 。 are plotted in the right-hand panel.

code of Gratton \& Sneden (1990) and a subsample of stars with the highest S/N spectra.

\section{Results}

Fig. 1 shows the derived $\mathrm{Li}$ abundances vs. $\mathrm{T}_{\text {eff }}$ for IC 2602 and IC 2391 . The derived metallicities for the two clusters are $[\mathrm{Fe} / \mathrm{H}]=-0.03 \pm 0.01$ and $-0.0 \pm 0.03$, respectively, i.e. they are nearly solar and comparable to that of the Pleiades.

The comparison of the Li EWs and Li abundances for IC 2602 and IC 2391 stars with the Pleiades (Fig. 2) confirms the previous finding by Randich et al. (1997) that late G and early $\mathrm{K}$ stars in IC 2602 already present a star to star scatter in Li abundances similar to, albeit not as large as the one in the Pleiades. A scatter is also seen among late-K and $\mathrm{M}$ dwarfs. This indicates that the scatter is already present at $\sim 50 \mathrm{Myr}$ and must develop during the PMS. Unlike in the Pleiades, the scatter seen in IC 2602 shows no evident correlation with rotation. IC 2391 presents much less scatter than IC 2602, suggesting that the amount of scatter at any given age may depend on the individual cluster and possibly on different initial conditions.

Stars more massive than $\sim 1 M_{\odot}$ show no sign of depletion in both clusters, while cooler stars are all lithium depleted, with the amount of depletion increasing to cooler temperatures. The distribution of $\mathrm{Li}$ abundances vs. $T_{e f f}$ 
is similar for the two IC clusters down to $\sim 5000 \mathrm{~K}$, but at lower temperatures the stars of IC 2391 tend to have less lithium than the stars of IC 2602 of similar temperature, i.e. the drop-off of lithium towards lower masses appears to start at an earlier color in IC 2391 than in IC 2602. This could suggest that IC 2391 is somewhat older than IC 2602, as recently proposed by Barrado et al. (1999) using the Li depletion boundary method, but this conclusion would hold only if lithium depletion is produced by standard mixing mechanisms (e.g. Pinsonneault 1997).

G and early K stars in IC 2602 are, on average, somewhat more lithium rich than their counterparts in the Pleiades. However the coolest stars in IC 2602 (and a fortiori in IC 2391) appear as depleted as the lowest lithium stars in the Pleiades. At the age of the Pleiades, stars in IC 2602 and IC 2391 are expected therefore to be more lithium depleted than stars of the same mass in the Pleiades. This suggests that lithium depletion might not be a single function of age for all clusters.

\section{References}

Barrado y Navascués, D., Stauffer, J.R., \& Patten, B.M. 1999, ApJ, in press Bessel, M.S. 1991, AJ 101, 662

Carlsson, M., Rutten, R.J., Bruls, J.H.M.J., \& Shchukina, N.G. 1994, A\&A 288, 860

Gratton, R.G., \& Sneden, C. 1990, A\&A 234, 366

Jeffries, R.J. 1999, in Stellar Clusters and Associations: Convection, Rotation, and Dynamos, eds. R. Pallavicini, G. Micela, \& S. Sciortino, ASP Conf. Ser., in press

Pasquini, P. 2000, these Proceedings

Pinsonneault, M.H. 1997, ARA\&A 35, 557

Randich, S., Aharpour, N., Pallavicini, R., Prosser, C.F., \& Stauffer, J.R. 1997, A\&A 323, 86

Randich, S., Pallavicini, R., Meola, G., Stauffer, J.R., \& S.C. Balachandran 2000, A\&A, submitted

Soderblom, D.R., Jones, B.F., Balachandran, S., Stauffer, J.R., Duncan, D.K., Fedele, S.B., \& Hudon, J.D. 1993, AJ 106, 1059

Stauffer, J.R., Hartmann, L.W., Jones, B.F., \& McNamara, B. 1989, ApJ 342, 285

Stauffer, J.R., Hartmann, L.W., Prosser, C.F., Randich, S., Balachandran, S., Patten, B.M., Simon, T., \& Giampapa, M. 1997, ApJ 479, 776 